\title{
L'evocazione del reale tra pittura e scrittura negli esordi narrativi di Carlo Dossi
}

\author{
Linda GAROSI \\ Área de Filología Italiana \\ Universidad de Córdoba (Spain) \\ linda.garosi@uco.es
}

\begin{abstract}
RiASSUNTO
L'opera narrativa dello scapigliato Carlo Dossi stabilisce, fin dall'inizio, un vincolo di unione stretto tra diversi linguaggi artistici. Nel comune slancio verso un rinnovamento espressivo che contraddistingue la scena culturale italiana postrisorgimentale, la commistione tra parola e pittura indica a Dossi la via di un possibile allargamento dei confini della letteratura nella riformulazione della realtà. Nei suoi primi scritti la lingua conferisce alla pagina un carattere iconico, figurativo. Le soluzioni stilistiche approntate da Dossi consentono una resa visiva della percezione del mondo che risponde, in modo affatto originale, all'esigenza di sintesi tra rappresentazione oggettiva ed evocazione memoriale. L'analisi di alcuni luoghi testuali significativi è corredata da dati contestuali che testimoniano la consapevolezza con cui lo scrittore opera e i referenti da cui trae le maggiori suggestioni.
\end{abstract}

Parole chiave: Carlo Dossi, romanzo italiano dell'Ottocento, pittura e letteratura, scapigliati, macchiaioli.

[Recibido, septiembre 2014; aprobado, diciembre 2014]

La evocación de lo real entre la pintura y la narrativa en el debut de Carlo Dossi

\begin{abstract}
RESUMEN
La obra narrativa del scapigliato Carlo Dossi establece, desde sus comienzos, un nexo de unión fundamental entre distintos lenguajes estéticos. En el impulso común para la renovación expresiva que caracteriza la escena cultural italiana postrisorgimentale, la fusión entre la palabra y la imagen indica a Dossi el camino para ensanchar los límites de la literatura en la exploración de lo real. En su primera novela L'Altrieri, la lengua otorga a la página un carácter icónico, figurativo. Las soluciones estilísticas elaboradas por Dossi llevan a una representación de cariz visivo de la percepción del mundo que responde, de una forma completamente original, a la necesidad de síntesis entre representación objetiva y evocación autobiográfica. El análisis de algunos fragmentos significativos del texto va acompañado por datos contextuales, más o menos conocidos, que corroboran la conciencia con la que el escritor actua así como los referentes a los que mayormente se inspira.
\end{abstract}

Palabras clave: Carlo Dossi, novela italiana de la segunda mitad del siglo XIX, pintura y literatura, scapigliati, macchiaioli. 


\section{Introducción}

Nella scena culturale italiana degli anni Sessanta dell'Ottocento, le novità pittoriche rivestono un ruolo di primo piano nei dibattiti che, prima a Firenze e poi a Milano, si accendono sull'urgenza di un rinnovamento di tutta l'arte italiana. Ne costituiscono di fatto la spinta decisiva dando l'abbrivo non solo a una vivace riflessione teorica, bensì propiziando $\mathrm{i}$ primi - ed incerti - tentativi di commistione tra letteratura, arti visive e musica (Bigazzi 1969: 53). In tale terreno affondano le radici di quel connubio tra linguaggi e stili artistici tipico della Scapigliatura. Particolarmente proficue si riveleranno le suggestioni che Carlo Dossi trae dalle inedite maniere pittoriche con cui, nel capoluogo milanese, si assimilano e rielaborano le tecniche dei macchiaoli fiorentini. Le note qui proposte intendono soffermarsi sull'incidenza, già segnalata dagli autorevoli studi di Isella (1958) e di Mariani (1967), che le sperimentazioni pittoriche ebbero nella fase aurorale dell'allestimento di un nuovo linguaggio narrativo. Dossi pare intuire, nell'intersecarsi di diverse modalità di rappresentazione artistica, l'opportunità di un allargamento dei confini stessi della letteratura grazie al quale poter soddisfare il bisogno di aderenza al 'vero' evitando, nel contempo, il mimetismo prodotto dalla pedissequa imitazione della realtà. Va precisato con Bigazzi che «Dossi guarda al mondo com'è, il suo crivello, cioè lo stile, non intende trasferire nella realtà i propri sentimenti ma far esplodere l'evidenza di oggetti e figure per mezzo del termine linguistico adoperato» (1969: 177). D'altro canto, l'intenzione iconica, che soggiace alle nuove soluzioni espressive allestite dal giovane autore, non si manifesta solo in senso espressionistico come segnalano Bigazzi o Isella, ma anche in senso impressionistico, dal momento che è funzionale alla resa dell'immagine della realtà così com'è percepita dal soggetto-artista (Portinari 2004; Patrizi 2009). Il che è esemplificato perlopiù in quelle pagine dedicate alla rievocazione del passato in chiave autobiografica: la parola che dà corpo alla visione soggettiva del mondo costituisce la sintesi tra oggetto e soggetto, tra forma e contenuto. Gli effetti di 'visività' dell'opera dossiana poggiano sull'equilibrio raggiunto tra la realtà fenomenica esperita dall'io e la narrazione che la ricrea artisticamente ${ }^{1}$.

\section{Il cammino parallelo delle arti}

\subsection{Elementi contestuali}

Più in generale, per quanto riguarda il fenomeno scapigliato a cui è legato il nome di Dossi, è noto che la visione unitaria delle arti costituisce il tratto distintivo e comune di personalità artistiche fortemente ecclettiche. Non è casuale che, nei primi e più autorevoli studi dedicati alla Scapigliatura, si tratteggi la fisionomia letteraria del gruppo richiamando l'idea di «arte totale» quale fondamentale nesso di coesione tra i suoi esponenti (Mariani 1967; Nardi 1968). Le parole di un testimone d'eccezione ricordano, inoltre, che nei cenacoli milanesi frequentati da chi sfidava la cultura ufficiale, le arti si riunivano «spesso nelle tendenze, non di rado nelle persone» (Levi 1913: 9). Ciò può essere letto come la concretizzazione dell'idea programmaticamente espressa da Giuseppe Rovani, il quale, rinvenendo nell'irresistibile progresso della civilità umana il «simultaneo cammino delle Tre

\footnotetext{
${ }^{1}$ Dossi afferma nella Nota azzurra 2232: «il poeta oggidì non scrive più ciò che vede, ma ciò che veduto ripensa. E il lettore non sente più ciò che legge, ma ciò che letto riflette. Così la natura non ci arriva se non di terza mano» (Dossi 1968: 141). Queste parole, va detto, definiscono la sua visione della letteratura umoristica.
} 
Arti sorelle» (1874: V), fu il primo a teorizzarne la vicinanza. Tuttavia, va detto che, al di là della maggiore o minor rilevanza che tale teoria dovette assumere, l'abitudine alla frequentazione di diversi ambiti estetici di molti protagonisti della scena 'eversiva' milanese portò a forme di commistione che infrangevano la categorica separazione fissata dal critico ${ }^{2}$. I continui scambi e i contatti intensamente vissuti (si pensi, tra gli altri, a un Emilio Praga pittore e poeta o a un Arrigo Boito poeta, narratore e compositore) fornivano spunti di riflessione e nel contempo stimolavano forme di ibridismi che, seppur appena abbozzate, rispondevano alla volontà di un ampio progetto di aggiornamento espressivo. Che tale dinamica sembri anticipare quella linea maestra lungo la quale fioriranno le esperienze delle avanguardie novecentesche è evidente, tuttavia si tradusse per lo più in dichiarazioni e in operazioni formali non del tutto privi di certo velleitarismo ${ }^{3}$. In ogni modo, tra i vari tentativi di ibridismo non mancarono soluzioni espressive che, al di là della loro singolarità, paiono precorritori. Al riguardo, e tra le voci più recenti, Patrizi afferma che «il caso di Carlo Dossi (1849-1910) è sintomatico di quel passaggio cruciale di poetiche, filosofie del linguaggio e teorie estetiche, in cui vanno collocate le prime tracce del lungo cammino novecentesco verso la contaminazione dei linguaggi estetici» (2009: 31). Non si intende qui entrare nel merito del dibattito quanto piuttosto, d'accordo con le premesse precedentemente formulate, collocare la propensione pittorica della penna dell'autore all'interno di specifiche coordinate, al fine di definire la valenza culturale e storica di un'elezione personale dagli esiti stilistici originalissimi.

Va sottolineato che, se il raccordo tra le diverse istanze semiotiche è calibrato in modo esemplare nel romanzo più «umoristico» del nostro, La Desinenza in A (1878), colpisce tuttavia come, fin dai primi lavori, sia già presente nelle forme linguistiche che assume lo sperimentalismo stilistico la ricerca di effetti di tipo visivo ${ }^{4}$. Era ben definita, inoltre, la consapevolezza con cui l'autore, fin dagli esordi, chiama in causa la pittura additandola come fonte innegabile di ispirazione per la sua scrittura, o anzi meglio dire, per la messa a punto del suo progetto scritturale. La critica è d'accordo nell'affermare che l'atto germinale dell'invenzione espressionistica dossiana, nata prematuramente ma già compiutamente concretizzata nell'Altrieri e poi nella Vita di Alberto Pisani ${ }^{5}$, sia da vincolare strettamente all'attenzione con cui il giovane guarda al nuovo corso pittorico, in particolare quello

\footnotetext{
${ }^{2}$ Per un riepilogo del dibattito critico sul peso della «teoria delle tre arti» all'interno dell'eterogenea poetica scapigliata, si rimanda al saggio di Giovanna Scarsi, Scapigliatura e Novecento: poesia, pittura, musica (1979).

${ }^{3}$ Dal saggio di Tessari intitolato Scapigliatura. Un'avanguardia artistica nella società preindustriale (1975) in poi, è stata spesso affrontata dalla critica l'eventualità di assegnare alla Scapigliatura milanese la condizione di prima avanguardia artistico-letteraria italiana. A questo proposito, Roberto Carnero chiarisce che «se parlassimo di avanguardia tout court a proposito della Scapigliatura, rischieremmo di commettere un peccato di anacronismo. Ci troviamo - negli anni Sessanta dell'Ottocento - in un'epoca di transizione: in campo politico, sociale e culturale. E la Scapigliatura rappresenta un fenomeno ibrido, tipico di un momento storico di mutamenti radicali, i cui esiti però non è ancora dato intravedere nella loro compiutezza» (2007: 12).

${ }^{4} \mathrm{Nel} \mathrm{misurare} \mathrm{la} \mathrm{distanza} \mathrm{tra} \mathrm{La} \mathrm{Desinenza} \mathrm{in} \mathrm{A} \mathrm{e} \mathrm{L'Altrieri,} \mathrm{Isella} \mathrm{definisce} \mathrm{la} \mathrm{lingua} \mathrm{del} \mathrm{primo} \mathrm{romanzo} \mathrm{in} \mathrm{termini}$ squisitamente pittorici e dice «la sua pasta espressiva fatta di diversi ingredienti (lombardismi, toscanismi, parole arcaiche o rare, voci straniere, cultismi, invenzioni personali), giustapposti nella stessa pagina, anzi nello stesso periodo e frase, come tocchi di colore diversi» (1995b: XXXVII).

${ }^{5}$ Sono tuttora fondamentali le considerazioni svolte da Dante Isella nel capitolo intitolato «Nascita dell'espressionismo dossiano» (1958: 5-13).
} 
scapigliato che, a Milano, sviluppa le premesse estetiche e tecniche dei macchiaioli fiorentini approdando ad esiti originali.

L'interesse dell'autore cresce in un contesto che non solo è percorso dai discorsi che indagano sulla convergenza tra i linguaggi artistici, a cui si è accennato in precedenza, ma è contraddistinto altresì dai fitti contatti tra artisti operanti in diversi campi. È utile ricordare che in una Milano, ormai postrisorgimentale e protoindustriale, Dossi muoveva i primi passi nell'orbita delineata dai modi dell'anticonformismo, dell'antiaccademicismo e dell'eversismo artistico degli scapigliati, stringendo intense relazioni amicali con i maestri 'ribelli', tra cui Cletto Arrighi, Giuseppe Rovani o appunto l'ammiratissimo Tranquillo Cremona $^{6}$. Questi, va ricordato, è l'assiduo frequentatore di casa Pisani Dossi a cui l'autore incaricherà il proprio ritratto oltre a quelli del padre e del fratello.

\subsection{Il sodalizio con Cremona}

L'ambiente culturale in cui si muove e cresce il giovane narratore è ricostruito in una delle prime prove letterarie del narratore nella quale la vicinanza a determinate tendenze pittoriche è centrale non solo nella costruzione della storia. Il volumetto del 1867, intitolato con il verso dantesco Per me si va tra la perduta gente. Racconto, può essere letto come un resoconto della scena culturale milanese e considerato una sorta di cartina tornasole degli elementi presenti in quell'officina in cui l'autore stava forgiando il proprio progetto stilistico e narrativo alla ricerca di un'impronta tonale singolare. Nel testo si possono cogliere i primi virgulti di uno specifico ingrediente dello sperimentalismo dossiano, che porta il narratore a tentare la fusione delle arti (nello specifico con la pittura) mediante la scrittura, ai fini di una ridefinizione, di un allargamento dei confini della letteratura stessa.

Nel ricreare la figura del protagonista, Dossi trae lo spunto iniziale dalle vicissitudini di Tranquillo Cremona. Con le vicende del pittore Roberto Marini ripercorre la biografia dell'amico allontanandosene solo nella parte finale. In più, è evidente, come sottolinea Montefoschi, che il personaggio fittizio porta con sé un bagaglio di concezioni estetiche e di tecniche pittoriche che ricalcano da vicino quelle di Cremona (1994: 36-41). Quando Marini dipinge il suo primo quadro da esporre, suscita l'aspro rimprovero del professor Carpaccio (figura in cui si addombra l'insegnante dell'Accademia milanese di Cremona, Giuseppe Bertini, pittore di genere). Questi, osservando con attenzione il lavoro del discepolo, «scoprì che il nostro Roberto si metteva per una strada cattiva tanto nella scelta del soggetto quanto nel sistema meccanico del trattarlo [...] Senza contorni! Diavolo! Senza contorni?... Bambagia, dunque, bambagia?» $\left(\operatorname{Per} m e\right.$, p. 190) ${ }^{7}$. Dossi attribuisce al suo Marini il rivoluzionario modo pittorico dell'amico Cremona, dimostrando di aver assimilato

\footnotetext{
${ }^{6}$ Un Dossi poco più che liceale, ma già animato da aspirazioni, veniva a contatto con quell'ambiente culturale milanese grazie alla frequentazione da parte di certi intellettuali del salotto della aristocratica famiglia Pisani Dossi, ma grazie anche all'amicizia con il giovane Luigi Pirelli che lo introduce nei circoli bohémien milanesi e con il quale scrive il primo racconto Educazione pretina e mette in cantiere la rivista Palestra letteraria. Per un approfondimento si veda la documentata introduzione di Paola Montefoschi all'edizione dei primi racconti pubblicati dall'autore. Anche il saggio celebrativo di Gian Pietro Lucini, L'ora topica di Carlo Dossi (1911) fornisce una preziosa ricostruzione delle relazioni strette dal giovane.

${ }^{7}$ Il testo di Per me si va tra la perduta gente. Racconto (Milano, tipografia Lombardi, 1867) è citato da Carlo Dossi, Due racconti giovanili (1994) con l'introduzione di Paola Montefoschi a cui si è fatto riferimento più volte.
} 
profondamente l'innovazione attuata da un pittore «della scuola dell'avvenire» nei suoi più celebri dipinti, dal Marco Polo, ai Due cugini al Falconiere, per citarne alcuni. Presta al suo personaggio la tecnica del 'non-finito', fatta di chiazze di luce, di macchie di colore sfumato, di contorni non delineati. L'autore novello dimostra di possedere una sensibilità figurativa privilegiata mostrando di conoscere nel dettaglio, sul piano dell'esecuzione, uno stile volto a sgretolare la materialità del «nudo realismo» e che non intende ricreare l'immagine della realtà con minuzia di particolari, ma restituirne l'ideale «moto dell'anima».

Meno lampante, ma di certo oltremodo significativo ai fini del presente discorso, è il fatto che lo scrittore non si contenti della mera adesione formale agli inediti procedimenti espressivi collaudati dal pittore scapigliato, ma piuttosto si avventuri a saggiare, coi modi esteriori del primo «vagito letterario», la fusione tra «quelle arti sorelle» il cui cammino simultaneo aveva additato Rovani. In questo senso è prezioso il riscontro, fornito da Montefoschi, di una notizia riportata da Luigi Primo Levi secondo la quale il soggetto della grande tela dipinta da Marini corrisponderebbe a uno dei temi storici che Cremona progettava di dipingere e che rimasero irrealizzati. La studiosa chiarisce che «oltre ai già noti Manzoni ai giardini pubblici e Vittorio Emanuele e Radetzki, vi era lo studio di un dipinto intitolato Bonifacio VIII e Sciarra Colonna: esattamente l'episodio rappresentato nel quadro "storico" dell'esordiente Marini» e aggiunge ${ }^{8}$.

\begin{abstract}
doveva trattarsi [...] di un progetto di cui il pittore aveva a lungo e dettagliamente parlato al giovane compagno scrittore [...] A sua volta Dossi, sostituendo la penna al pennello, trasferiva sulla pagina scritta le intenzioni dell'amico, ricreando nel proprio racconto il quadro irrealizzato, esattamente nella maniera in cui lo avrebbe dipinto Cremona, fedele non solo nella composizione della scena, nella disposizione e negli atteggiamenti dei personaggi [...] ma nella tecnica, nello stile, nello spirito [...] Insomma Dossi che aveva imparato a scrivere nel modo in cui Cremona dipingeva e andava affinando il proprio espressionismo al contatto con le altre sperimentazioni, aveva "dipinto" il quadro al posto dell'amico, in perfetta sintonia con le sue ragioni, assumendone la tecnica, tentando di approssimarsi alla maniera inimitabile con cui egli sapeva travasare nell'arte la vita [...] Questo mescolarsi delle esperienze e questa corrispondenza di intenti rappresentano, a nostro avviso, una delle più straordinarie sorprese che si celano tra le righe delle acerbe pagine giovanili del futuro grande scrittore (1994: 4041).
\end{abstract}

Tale rinvenimento è il segnale di procedure tecniche destinate a evolversi nella costruzione di una particolare visione della realtà mediante il linguaggio letterario. È utile aggiungere una considerazione che illumina, a nostro avviso, una linea di continuità tra il racconto e L'Altrieri. Nello stesso modo in cui l'autore riprende il terzo verso del Canto III dell'Inferno come preambolo di un repertorio di temi di stretta attualità letteraria, coniugando antico e moderno secondo una prassi strategicamente attuata, tra gli altri, anche da Cremona, è ipotizzabile che il narratore non si fosse scordato della lezione virgiliana del «visibile parlare» riportata nel Canto X del Purgatorio (v. 95). La paradossale affermazione può aver acquistato forza nell'intersezione con i coevi dibattiti sulle proprietà conoscitive dell'arte figurativa nonché sul reale e ideale in arte $^{9}$, mostrando al giovane come la fusione

\footnotetext{
${ }^{8}$ La studiosa riporta l'informazioni data da uno dei primi critici e biografi di Tranquillo Cremona Luigi Primo Levi (1913: 45), e ripresa a sua volta da Giorgio Nicodemi (Tranquillo Cremona, Milano, Mondadori, 1933: 81).

${ }^{9}$ Patrizi ricorda un brano di uno scritto di Vittorio Imbriani raccolto in Critica d'arte e prose narrative (1868) in cui si definisce la 'macchia' come «il ritratto della prima impressione lontana di un oggetto ovvero di una scena;
} 
tra pittura e parola consentisse di plasmare verbalmente immagini consustanzialmente reali. Nel tentativo di rinnovare il linguaggio narrativo, l'intuizione spingerà Dossi non tanto verso la via della descrizione ecfrastica di stampo classicista, quanto verso soluzioni espressive che conferiranno alla sua pagina un carattere di «prensilità visiva». È possibile così andare oltre i limiti del nudo oggettivismo senza che, per questo motivo, venga intaccato il ruolo prioritario conferito dalle nuove tendenze artistiche al vedere, all'occhio, quale privilegiato strumento conoscitivo del mondo ${ }^{10}$. Alla similitudine dunque subentra l'immagine. Di conseguenza, nella ricerca di un linguaggio narrativo proprio, Dossi si rivolge a stili espressivi inediti da cui tenta di assimilare procedure diverse dalle quali partire per «ritrovare una forza specifica della lingua, una sua possibilità di affermarsi come dizione assoluta del mondo» (Patrizi 2009: 34). Allo scarto della razionalità trionfante è funzionale un approccio alle arti visive quali testimoni di una realtà noumenica urgente dietro la fenomenicità quotidiana. Ma questa è già una dialettica tipica dell'avanguardia. Dossi pare piuttosto voler piegare la lezione classica del graphice scribere alla volontà di dare l'illusione di veracità alla ricreazione artistica del mondo. Non perde quindi di vista la materialità stessa della scrittura; da cui il potere evocativo del segno linguistico. Se la penna è assimilata al pennello, è anche perché la calligrafia (il nero su bianco) era in origine, con il geroglifico, unione di «plastica e pittura». Su tale base, la lingua e l'immagine hanno conservato proprietà comuni il cui nesso indissolubile viene garantito dalla mediazione della scrittura ${ }^{11}$. Di ciò è significativo uno dei numerosi interventi del Dossi teorizzatore. Nella Nota azzurra 5178 , che potrebbe fungere senz'altro da postilla alle affermazioni rovaniane sulla «teoria delle tre arti», si legge:

fra le prove della fraternità delle tre arti (letteraria, figurativa, musicale) ce n'è una storica. La scrittura, espressione dell'arte letteraria, era in origine plastica e pittura (la scrittura geroglifica o letteratura rebus). Piú tardi, la scrittura rappresentò, invece del segno, il suono (scrittura fonetica) donde il legame colla musica - la scrittura è la più antica delle arti plastiche. (Dossi 1964: 721)

\section{Nell'officina dello scrittore}

Sorprendente è il viraggio compiuto dalla penna dossiana appena un anno dopo con l'opera intitolata L'Altrieri e sottotitolata Nero su bianco ${ }^{12}$. Tale passaggio è segnato, tra

l'effetto primo e caratteristico che s'imprime nell'occhio dell'artista che vegga l'oggetto o la scena materialmente, sia che percepisca questa o quello con la fantasia o a memoria [...] L'eseguire, il terminare un quadro non è altro che un ravvicinarsi sempre più all'oggetto; che sbrogliare e fissare ciò che ci è passato sugli occhi come un barbaglio» (2009: 35).

${ }^{10}$ Nell'opera dossiana la modalità fondamentale di accesso al mondo da parte dell'io dell'artista «passa per l'occhio» (Portinari 2004: 3).

${ }^{11}$ Illuminanti sono le considerazioni con cui Giovanni Pozzi delinea la realtà sostanziale dell'iconismo poetico. Dice il critico che «le proprietà che lingua e disegno hanno in comune dipendono dal fatto che ambedue sono entità quantificate. L'iconismo in poesia si produce da quando le marche della quantità, quelle della lingua e quelle del disegno, si congiungono a creare un solo effetto; da quando cioè le quantità linguistiche divengono linee, superfici, posizioni, tagli, cornici. Ciò avviene con la mediazione della scrittura» (1996: 29).

${ }^{12}$ L'Altrieri. Nero su bianco esce a Milano nel 1868 e su questo testo verterà l'analisi qui proposta dal momento che ci interessa cogliere i primi riverberi delle tendenze figurative scapigliate sulla prosa narrativa di Dossi. Si ricordi che una seconda edizione interamente riveduta esce in quattordici appendici sulla rivista romana La Riforma tra il 24 maggio e il 9 giugno 1881; mentre la terza edizione, il cui testo ripropone quello apparso in rivista, esce sempre a Roma con una prefazione intitolata «Agli scrittori novellini» presso lo Stabilimento Tipografico Italiano nel 1881. 
l'altro, da un mutamento nell'approccio al dialogo fra le arti. Nel racconto si registra una prossimità tematica motivata dalla trasposizione letteraria dell'amicizia tra Dossi e Cremona e a cui si legano gli architesti figurativi con cui la narrazione istaura legami di tipo intertestuale. Nell'Altrieri le nuove tendenze figurative non fungono più da soggetto della narrazione, ma calano a fondo nel tramarsi di un linguaggio narrativo dalla cifra stilistica originalissima. L'autore lombardo vi fa sfoggio di una gran sicurezza nel maneggiare una lingua oscura e indisciplinata usata da un narratore omodiegetico per riesumare l'infanzia perduta. A detta di Isella il «recupero memoriale» messo in atto da Dossi doveva portarlo, con largo anticipo sui simbolisti francesi, alla «scoperta, per proprio conto [...] del valore evocativo della parola», grazie tra l'altro al fatto che a costui, «amico di Cremona e di Ranzoni, ammiratore di Grandi, gli stimoli e i suggerimenti più giusti gli dovevano venire dalle sperimentazioni figurative di cui era osservatore appassionato» (1995a: XXIII). Già la critica contemporanea ebbe a segnalarlo. In una recensione alla versione romana dell'Altrieri, quella del 1881, Luigi Capuana osservava che, leggendo una pagina di Dossi, «si prova lo stesso effetto che innanzi un quadro del Cremona» (1882: 68). L'autore stesso aveva confessato in una lettera allo zio Alberto Quinterio, datata 1869 e a lungo rimasta inedita, il debito contratto nei confronti di Cremona, affermando di aver imparato a scrivere «non dai polverosi scartafaci cari all'abate Cesari [...] ma da lui, dal suo pennello, riboccante di sole e di amore, sàturo di finezze, di sapienza, di originalità» (Isella 1988: XII-XIII) ${ }^{13}$. Queste identiche parole si ritrovano in esergo all'edizione romana della Desinenza in A. Conviene chiarire brevemente che l'epigrafe, in quella sede, funge più che altro da affettuoso ossequio all'amico e maestro di un tempo che non da dichiarazione di intenti concretizzatisi nell'opera dedicata, «testo che rinvia, invece, in forma citazionale o allusiva» osserva Saccone «ad un altro universo figurativo, quello allestito dalle composizioni hogarthiane» (1995: 83).

Come si è detto in precedenza, ed è utile ricordare, negli anni della formazione e della stesura dei primi romanzi, il giovane scrittore lombardo doveva aver ben presente le idee espresse dall'apprezzatissimo Giuseppe Rovani sulle «mutue rispondenze», i percorsi paralleli tra le diverse modalità estetiche. Negli scritti raccolti prima nella Storia delle lettere e delle arti in Italia (1855-58) e poi rifusi nel volume Le tre arti del 1874, Rovani tracciava i contorni di una visione unitaria delle arti nella storia, mettendo però in guardia da ogni forma di compenetrazione poiché «ogni qual volta un'arte vuole invadere un campo non suo, necessariamente si snatura e si fa ibrida, smarrendo i propri attributi più sostanziali nel tentativo di raggiungere gli altrui» (1874: VI). Tali affermazioni dovevano essere sì calate in profondità nel pensiero dello scrittore, ma di fatto vennero poi indirizzate da questi verso ben altra direzione, come dimostra la lettura attenta del testo in cui Dossi recensisce La giovinezza di Giulio Cesare (1873). Il Cenno, raccolto in Fricassea critica (1906), offre infatti un giudizio che, benché sia ampiamente favorevole alla fattura di quel romanzo

Nel presente articolo si seguirà il testo della prima versione e si cita da: Carlo Dossi, Opere, a cura di Dante Isella, Milano, Adelphi, 1995.

${ }^{13}$ Isella ne riporta alcuni brani nella nota introduttiva della sua edizione del 1988 dell'Altrieri. I passaggi trascritti dal critico sono significativi giacché dimostrano fino a che punto l'autore avesse consapevolezza piena dei procedimenti messi in atto nell'elaborare il proprio pastiche letterario, risultato questo, e Isella cita da Dossi, «dell'inimicizia per le frasi, per le metàfore note di troppo» e della volontà di non conformarsi alla «monotona musica» della letteratura di moda (p. XII). 
storico, ricorre a termini che sembrerebbero riferirsi piuttosto all'iconismo della pagina dossiana; in particolare quando sottolinea che «in questo libro men si discute o dimostra che non si descriva o a meglio dire si pinga. E appunto è una serie di scene, che sono il trionfo di quella preziosa virtù che i latini chiamavano graphice scribere» (Dossi 1995: 1408). Allo scopo di esaltare gli effetti di ordinata plasticità dell'opera, volti a soddisfare l'esigenza, particolarmente sentita dagli artisti più innovatori, di aderenza al reale, di fedeltà all'«odierno», Dossi richiama una lunga tradizione letteraria non in termini puristi, ma in quelli aggiornati sulle linee programmatiche tracciate dal vivace dibattito a lui coevo sul realismo in arte. Così facendo, però, cambia di fatto le carte del maestro lasciando trapelare il proprio operare con oculata sottigliezza. Se Rovani fissa un parallelismo tra le arti basato sulla comunanza di intenti delegittimando allo stesso tempo qualsiasi forma di sconfinamenti, Dossi, è noto, non disdegna affatto il compenetrarsi fra forme d'arte diverse. Anzi, la complessità di tale dialogo agisce da stimolo all'approccio personale alla letteratura: su tentativi di ibridismi e di miscelazioni spesso definisce le sue scelte stilistiche, le sue costruzioni sintattiche e l'impianto strutturale stesso della narrazione. Le considerazioni dossiane sul libro di Rovani divengono, quindi, particolarmente suggestive se riferite all'importanza che la commistione tra scrittura e pittura assume nell'innovazione messa in atto dal giovane scapigliato.

Il senso di tale attenzione, come pure alcune importanti indicazioni operative, emerge esplicitamente in un altro famoso scritto che, nello stesso anno, l'autore dedica a Cremona in occasione dell'Esposizione di Belle Arti a Brera. Qui Dossi premette alla descrizione del quadro esposto dal pittore, Silenzio amoroso, il motivo fondante dell'ammirazione verso chi considerava un «insaziabile amante del poetico vero» (1995: 1283). Condivide la scelta estetica dell'autore di rendere «l'idea generica di una passione umana» non mediante la minuziosa «illustrazione di un fatto particolare» tesa all' «imitazione del vero», ma impiegando la non finitezza. Osserva, inoltre, che nei suoi quadri non ci sono «marionette orlate di nero e di colore ripiene, profili che sembran confini di carte geografiche e dividono anime [...] in esse, non colori, non tela, ma persone, ma affetti» (1995: 1284-85).

Sulla base di siffatta comunanza di intenti artistici, lo scrittore, nonché certa «consanguineità» di mezzi espressivi, porta a un elevato grado di affinità l'impasto della pagina dossiana e la tela dell'amico, soprattutto per quanto riguarda il romanzo qui oggetto di analisi. Prendendo in esame L'Altrieri si può notare con Sacco che «l'opera preferisce assomigliare alla tela di un pittore, ad una partitura musicale muovendosi in direzione di quella fusione fra le diverse forme di espressione artistica spesso teorizzata da Dossi» (1996: XX). Il proprio autore esplicita tale nesso nella Nota azzurra 2382 in cui si legge "L"“Altrieri" si compone di 3 parti [...] D. avea tentato in questa $3^{\text {a }}$ parte, non di far sentire le parole, ma $\mathrm{i}$ suoni; di avvicinarsi cioè, il più che gli fosse stato possibile, alla musica; come avea tentato nella parte seconda di fare più che non letteratura, pittura» (Dossi 1964: 188-189). Dell'evidenza di tale somiglianza si fa portavoce altresì Luigi Primo Levi. Il critico e biografo riflette sul fatto che la novità della proposta artistica avanzata da Tranquillo Cremona giunge ad irritare lo spettatore contemporaneo che:

abituato a ricevere percezioni facili, distinte, lineari, contornate; abituato perciò a ritenere la maniera dei quadri che gliele dava come l'unica espressione pittorica del vero, s'inalberò e rimase incerto, confuso quando non vide più dinanzi a sé [...] la vernice, la levigatezza, la lucidezza solite, abituali. (Levi 1913: 27) 
Una reazione simile, e per ragioni analoghe, è suscitata dalla prosa narrativa di Dossi nel pubblico di suoi lettori e critici a causa dell'oscurità del suo stile. Conviene ricordare, inoltre, che l'intreccio tra arti diverse è funzionale alla disgregazione del linguaggio narrativo tradizionale. La prosa di Dossi è innervata da una forza centrifuga che tende inequivocabilmente alla "dispersione», a una assenza di consequenzialità, palesi sia nelle soluzioni linguistiche sia nell'impianto narrativo. Detto ciò è facile capire fino a che punto risultasse suggestivo l'ideale cremoniano della 'non finitezza' (e come lo sarebbe stato in seguito quello della «linea serpentina» formulato dal pittore inglese Hogarth nel saggio Analysis of Beauty del 1753).

\section{Effetti di tipo visivo nell' Altrieri}

\subsection{Soluzioni formali}

La vocazione pittorica che sottende la scrittura di Dossi si traduce in un uso della materia verbale che attribuisce alla pagina un'efficacia visiva. Se il pittore ha disposizione i colori della sua tavolozza e li stende con tocchi rapidi sulla tela accostandoli abilmente tra di loro ad ottenere un cromatismo chiaroscurale, Dossi si avvale di soluzioni raffinatissime d'elaborazione formale che puntano ad una analoga restituzione della realtà. Va ricordato, ancora una volta, che i tratti costitutivi dello stile pittorico con cui la tessitura prosistica dell'Altrieri viene a stabilire analogie d'esecuzione sono «la macchia cromatica, la labilità ectoplasmatica dei contorni, il luminismo sfumato fino alla dissolvenza» (Saccone 1995: 81). A Milano i principali interpreti della pittura antiaccademicista accoglievano sí i modi operativi dei Macchiaoli fiorentini, ma ne accentuavano i valori evocativi della rappresentazione figurativa ${ }^{14}$.

È lecito interrogarsi su quali siano i modi e i mezzi formali di cui si avvale Dossi per piegare la lingua a una resa della visione della realtà dagli effetti impressionisti. Su tale fronte, sono note le osservazioni di Isella riguardanti la qualità pittorica derivante dall'eccleticismo delle fonti a cui attinge lo scrittore nell'elaborazione di un impasto linguistico motivato «in funzione del delicato recupero di una propria memoria, prensile, adatta a fermare sensazioni e ricordi sfuggenti, nella vaghezza di ogni passato e dei suoi contorni sfumati, con vive, intense macchie cromatiche» (Isella 1995b: XXXVII). Le «alchimie lessicali» e l'ampia gamma di tropi non costituiscono gli unici mezzi a disposizione di Dossi nella restituzione impressionistica del mondo. Va infatti messo in rilievo il periodare sincopato che, seppur finalizzato alla restituzione dell'urto emotivo con cui affiorano i ricordi nella mente dell'artista, si basa su costruzioni della frase e del periodo in grado di creare un'immagine ad alta intensità iconica e dalle innegabili assonanze con le contemporanee esperienze raffigurative degli scapigliati. Già Mariani aveva osservato che mediante procedimenti sintattici volti alternativamente all'accumulo o alla condensazione viene verbalizza-

\footnotetext{
${ }^{14}$ I pittori scapigliati - tra cui ricordiamo Tranquillo Cremona, Giuseppe Grandi e Daniele Ranzoni- accolgono sì i modi approntati dall'aggiornamento tecnico compiuto dai toscani ma, nel contempo, li innestano nella tradizione romantica lombarda e ne depurano gli aspetti eversivi e di denuncia sociale, continuando invece la linea più formalistica, meno ideologizzata. Il realismo viene inteso come strumento di oggettività fine a se stesso. Spiega Adriano Cecioni che «il Soggetto è valido come pretesto per sciogliere un problema di ombre, di luce, di colore». La citazione da Adriano Cecioni, Scritti e ricordi, Firenze, Tipografia domenicana, 1905 è tratta da Bietoletti (2001: 19).
} 
ta l'evanescenza fortemente evocativa dei ritratti realizzati dal pittore scapigliato ${ }^{15}$. Il critico spiega che:

gli elementi della rappresentazione si allineano, non si compongono in un armonico insieme perché lo scrittore non si propone la composizione ma la scomposizione, la frantumazione del discorso e il conseguente isolamento della parola immagine o dell'espressione sintetica che racchiude quell'immagine alla quale egli vuol dare tutto il rilievo. (1967: 489)

Dall'attento vaglio della sintassi dossiana, Caputo, nel suo magistrale studio, ne isola i caratteri sintattici costanti e ne segnala «il gioco combinatorio fra segmenti lunghi-brevi, lunghissimi-brevissimi, fra sequenze a sviluppo lineare, prevalentemente paratattico, e sequenze involute, con forte scardinamento della successione normale di parole e proposizioni» (2000: 22). È frequente il ricorso a periodi lunghi costruiti con semplicità giocata sull'intreccio fra ipotassi e paratassi, per cui si ha «un andamento elencativo e paratattico, ma i nuclei, in cui risulta ripartito da una forte pausa interpuntiva [...] sono costituiti al loro interno da due o più subordinate» (p. 26). Si ottiene dunque un effetto «di forte messa in rilievo dei soggetti dei singoli blocchi», dal momento che tali sequenze verbali così costituite «permettono di dare evidenza a figure, oggetti, dettagli, tratto che rimanda a una delle caratteristiche dello stile nominale» (p. 26). Tali espedienti formali consentono di comprimere all'interno di una sequenza un cospicuo numero di «blocchi di soggetti» in funzione dell'affiorare subitaneo dei ricordi dalla forte pregnanza emotiva ma anche con effetti dirompenti di tipo raffigurativo. Inoltre, muovono nella direzione di una composizione della pagina fondata su di un accumularsi di elementi atto a creare, o meglio a evocare, uno scorcio di realtà dalla grande immediatezza visiva, se non addirittura scenica ${ }^{16}$.

Si legga a questo riguardo l'incipit del romanzo, quasi una soglia intratestuale, che ospita una riflessione in termini metaforici sull'atto di ripiegamento introspettivo che presiede la creazione artistica. L'affiorare momento e imprevedibile dei vividi ricordi dell'infanzia nella mente dell'io narratore è reso da uno stile scomposto che rompe la consequenzialità del discorso, mentre l'immagine è resa in modo frammentario:

I miei dolci ricordi! Allorachè mi trovo rincantucciato sotto la cappa del vasto camino, nella oscurità della stanza [...] l'anima mia, stanca di febrilmente tuffarsi in sogni di un lontano avvenire e stanca di battagliare con mille dubi, con le paure, con gli scoraggiamenti, stríngesi a un intenso melancónico desidério per ciò che fu. Io li evóco allora i mie' amati ricordi, io li vóglio; li vóglio, uno per uno, contare come la bisnonna fa de' suoi nipotini. Ma essi, in sulle prime, se ne tirano indietro: quatti

\footnotetext{
${ }^{15}$ Sull'effetto di pieno che caratterizza la prosa narrativa di Dossi, essendo l'enumerazione di cose una tecnica dall'indole squisitamente pittorica, osserva Isella «poche scritture saranno così dense di "oggetti" come qualunque pagina dell'Altrieri; ma in pochissime entreranno così scorporati della loro "oggettività"» (1958: 11).

${ }^{16}$ Estensibili alle soluzioni stilistiche approntate in tal senso da Dossi, e alle intenzione artistiche sottostanti, sono le parole di Pozzi che rischiarano gli elementi comuni tra lingua e disegno. Egli afferma che «la retorica ha pure preventivato figure isomorfiche di ogni tipo, lessicali, frastiche, periodali, quantitative e qualitative, le quali in particolari situazioni tematiche o affettive producono l'effetto opposto della variazione: isocoli, ripetizioni, enumerazioni, cumuli e via di seguito. Al di là delle motivazioni di forma e di contenuto, gli isocoli di ogni tipo costituiscono infallibilmente dei segmenti di materia linguistica di misura uguale e di uguale natura; in rapporto al testo totale quei blocchi uguali rappresentano delle divisioni che intersecano la massa totale. Poco ci vuole perché un'intenzione iconica vi si sovrapponga» (1996:30).
} 
quatti érano là sotto un bernóccolo del mio cipollone; io li annojo, li stúzzico; quindi, è buon diritto, se danno in capricci. Pure, a poco a poco, il groppo si disfa. (L'Altrieri, p. 5)

\title{
4.2. Qualche esempio dal testo
}

A tale proposito è utile portare al centro dell'analisi alcuni segmenti esemplificativi tratti da L'Altrieri. Nella prima parte («Lisa») si possono rinvenire brani più consoni a certe maniere pittoriche dell'impressionismo macchiaiolo originalmente accolto e sviluppato da Cremona. Le sensazioni ed emozioni vissute nella più tenera infanzia, tra cui quelle appartenenti all'idillio fanciullo tra Lisa e Guido Etelredi, sono evocate in sequenze in cui la rappresentazione della scena è resa con tratto fluido e trasparente. Il seguente frammento, di cui Isella offre una squisita analisi in chiave pittorico-musicale, è esemplificativo di alcuni mezzi tecnici adoperati secondo tale modalità espressiva. La frammentazione del periodo e il ritmo sincopato dell'eloquio, ottenuti dall'accumularsi di incisi, riproducono la discesa delle bolle di sapone raggiungendo un effetto figurativo simile a quello dato da rapidi pennellate:

\begin{abstract}
Allorachè ci penso, che bei tempi eran quelli! Quante volte io mi sento ancora a costa della mia piccola compagna, su quella ringhiera che rispondeva sopra la via, gonfiando bolle di sapone, le quali, staccátesi da la cannùccia (oh! le granate di casa) tremolávano, cullávansi nello spázio, poi, divenute colore cangiante, trasparentíssime - a gran dispetto di quattro o cinque ragazzi che le attendévano, la bocca aperta, curiosi - vanávano. (L'Altrieri, p. 18)
\end{abstract}

Il volo lento della bolla così come la sua lieve sospensione aerea sono resi mediante incidentali a funzione ritardante. La terna di verbi in frasi giustapposte viene chiusa dal verbo reggente in posizione di clausola, distanziato dunque dal soggetto quasi a esaltare il valore referenziale dell'elemento al centro del ricordo. Il mimetismo figurativo prodotto dalla costruzione sintattica viene accentuato altresì dalle notazioni luministiche $\mathrm{e}$ coloristiche.

Quando dalla prima parte dell'opera si passa alla seconda, «Panche di scuola», la rappresentazione della realtà sembra assumere altri connotati. Il movimento iniziale si chiude con la morte di Lisa che manda in frantumi il fragile mondo infantile del protagonista. Provoca uno «stracciamento di cuore», un disincanto tale che porta il bambino protagonista a non credere più nelle fiabe, a perdere l'innocenza edenica della prima fanciullezza. L'infanzia svanisce e lascia il posto alla scuola: un momento cruciale quanto difficile di crescita. Qui l'autore unisce alla restituzione del vissuto, la critica di una realtà sociale spiegando l'ampio arsenale del suo gusto satirico e grottesco. Si passa quindi dall'evanescenza adeguata ai toni eterei ed idilliaci con cui viene rappresentata l'infanzia del protagonista, alla densità di certe descrizioni ottenuta da una più compatta combinazione di ellissi-ipotassi variamente sovraccaricate da incidentali. Questa caratteristica stilisticoretorica è evidente nel racconto di anni in cui Guido getta le basi della propria personalità e cresce la consapevolezza di sé e del mondo che lo circonda. A parte l'impulso deformante che sottende queste pagine, la transcodificazione figurativa messa in atto (l'aveva ribadito lo stesso Dossi nella già citata Nota azzurra 2382) si traduce, come si diceva, in sequenze dalla forte condensazione di costrutti sintattici fino allo stile nominale. E allo stile nominale Dossi giunge in modo compiuto nella sequenza di presentazione dei compagni di classe del piccolo Guido: 


\begin{abstract}
Ed appancate, quante differenti testine! Là una ríccia siccome i trúciuoli del legnajuolo e castagnina chiara; quà, una arruffata, dal cavello aspro e castagnina oscura; presso, una bionda, a ciambelline, vera matassa di seta; poi, una nera, a gomma, lustra al par di uno stivale (se lustro) in séguito, tre cimate, una rossigna... E quanti diversi nasucci!... arricciati, a peperone, aguzzi, i più... incipienti... E quanti vispi occhiettini! grandicelesti, piccolineri, grisi che ammíccano, verdógnoli; quì, a lunghe cíglia, bassi come que’ di una mónaca; lì, strabuzzanti, da coccovéggia: o tondi come due-centésimi, o a sfenditura da caldarroste. (L'Altrieri, pp. 39-40)
\end{abstract}

La vivace e scoppiettante descrizione è anticipata da un'immagine sintetica (differenti testine) espressa da una esclamativa. Ne segue l'elenco dei particolari del volto (capelli, naso, occhi) a rendere, quasi impressionisticamente con rapidi e sicuri tocchi, il quadro d'insieme di un indisciplinato e variegato gruppo di bambini. La composizione dei soggetti nello spazio è guidato dalle coppie in opposizione di deittici (qua/la; qui/li; presso/poi). La coesione degli elementi della scena è garantita inoltre dalla ripresa anaforica, con variazioni, della struttura formata dall'aggettivo indefinito quanto e sostantivo (quante differenti testine!/ quanti diversi nasucci!/ E quanti vispi occhiettini!). Nello specifico di questo brano, i dispositivi «centripeti» creano un andamento concitato che viene a evocare la turbolenza del gruppo. Al di là della patina grottesca, quasi surreale, data dagli accostamenti inconsueti e stranianti proiettati dalle metafore, colpisce l'effetto cromatico delle capigliature e degli occhi, i quali vengono definiti mediante la nominalizzazione dell'aggettivo qualificativo a loro attribuito con un effetto del tutto simile a quello della rappresentazione pittorica dell'oggetto mediante macchie di colore.

Alla descrizione della scolaresca gruppo segue la presentazione di un altro personaggio, il maestro Ghioldi, lo zimbello dell'intera scolaresca e del preside. Il ritratto è rinchiuso in un inciso che scinde, allontanandoli, il soggetto, espresso da una metafora iperbolizzante ( $\mathrm{il}$ pettinatore morale di tutti questi meloni), dal proprio verbo in posizione di clausola ( $s i$ avanzò allora ver noi). L'incidentale è formata da tre nuclei di parentetiche grammaticalmente slegate che assume un'estensione tale da rovesciare il rapporto sintatticamente gerarchico con la proposizione in cui si inserisce. Qui la transcodificazione figurativa non è eseguita a livello semantico, ma esclusivamente a livello sintattico. La parentesi descrittiva mette a fuoco il nuovo personaggio, il soggetto dell'azione, con un efficace effetto teatrale del tutto paragonabile all'entrata di un attore su di un palconscenico. Di conseguenza l'amalgama di costrutti in inciso non restituisce solo una percezione visiva ma svolge una funzione presentativa:

\footnotetext{
il pettinatore morale di tutti questi meloni - un fuserágnolo malbailito, un po' scorretto di gambe, bírcio, senza un pelo al labro quantunque se lo carezzasse soventi e con un cinque o sei dozzine al più di capelli, tuttochè studiasse che la penna d'oca (in verità poggiata su di una molto visíbile orécchia) paréssegli ficcata nella capigliatura - si avanzò allora ver noi. (L'Altrieri, p. 40)
}

Al di là dell'intenzione deformante di quest'ultimo ritratto, entrambi i frammenti riportati sono rappresentativi di una strategia espressiva che contraddistingue, in particolar modo, il secondo capitolo del testo e che giunge a comporre, a tracciare, sulla pagina un'immagine del soggetto descritto visivamente corposa e cromaticamente densa ottenuta mediante la condensazione di elementi diversi, quanto eterogenei.

Accanto alle affinità espressive con il Cremona, ne rinveniamo una di tipo tematica, dato che i bambini sono uno dei soggetti preferiti dal pittore pavese. Una simile tangenza è rafforzata da una citazione intertestuale di un famoso quadro dell'artista, Il Falconiere. L'allusione all'opera precede il ritratto di un discolo compagno di scuola l'odiato Daniele Izar. È altresì l'indicazione metapoetica del rifiuto dell'autore della tradizione narrativa e 
della convenzionale divisione tra scuola antica e scuola odierna (a cui mette capo il Cremona). Nel presentare il ritratto del bimbo ebreo, Dossi offre così una chiave di lettura del tono caricaturale e grottesco che usa. Questo tono non è mai fine a se stesso, ma è funzionale alla rappresentazione della realtà che, appoggiata sulla forza d'urto della sovversione linguistica, restituisce la visione personale dell'autore atta a coglierne le contraddizioni, gli aspetti inediti. Si legga, in questa luce, il seguente brano:

\begin{abstract}
se adesso po' io vi presento questo Daniele come un marmócchio costruito coi gómbiti, con un viso da tromba, non crediate già che lo fáccia per convenzione, per quella brutta ruffiana che t'imbastisce in quattro gugliate un lavoro e che quì scrive: tiranno (moda antica) peloso più d'una cótica, occhi injettati di sangue, senti del guércio e zóppichi- o pure- tiranno (moda odierna) il Falconiere di Tranquillo Cremona- no, è puramente perchè va rispettata l'istoria» (L'Altrieri, p. 50)
\end{abstract}

\title{
5. Conclusioni
}

Per concludere, va ricordato che l'icononismo della pagina dossiana è da inquadrare in un ben preciso contesto culturale: quello dell'interesse degli scapigliati per le arti e dei dibattiti sul 'realismo'. La fusione tra linguaggio verbale e non verbale perseguita con procedimenti formali raffinatissimi è strumentale alla necessità di elaborare nuovi parametri espressivi e conoscitivi superando quelli che vengono avvertiti come i limiti della cultura del primo 'verismo, quello rappresentato da certo bozzettismo ottocentesco che metteva il referto documentario al centro della rappresentazione. Scrive Dossi nella lettera allo zio Quinterio del '68 «l'arte non è la natura. Essa, proprio, non cerca il vero, ma cerca che vero sembri l'imaginato... altrimenti, sarebbe una fredda, tediosa fotografia» (Isella 1988: XV). Nella restituzione artistica del dato reale l'autore pretende superare il canone di verosimiglianza e l'illusione della referenzialità, e ne rimanda un'immagine filtrata dalla propria visione personale. Da qui, allora, il fondamentale insegnamento di Cremona che segnala al giovane scrittore la direzione da prendere offrendogli la maniera inimitabile con cui egli sapeva travasare nell'arte la vita. È indicativo allora che Dossi, nel famoso articolo del 1873, rifletta sul fatto che il pittore riesca a restituire poeticamente la realtà operando una scelta su di essa e ritraendola «come dovrebbe essere»; l' «amante del poetico vero» deve «copiare direttamente sí il vero, ma nell'ambiente dell'animo proprio: deve, per così dire, stacciarlo attraverso il crivello del suo giudizio individuale» (Dossi 1995:1408). In questa ottica, l'autore indaga l'oggetto per farne esplodere la superficie in rapporto ad una visione soggettiva, quasi autoreferenziale. Difatti, sebbene Dossi cresca come scrittore nello stesso clima in cui si stava formando il verismo e la sua variante naturalistica, alle esigenze di rappresentazione del reale dà una soluzione espressionistica. Ne deriva un discorso narrativo che, nel rievocare la realtà, infrange l'omogeneità conferita all'immmagine del reale dall'impersonalità naturalistica. Si tratta di un'originale versione di realismo che è presentazione in chiave soggettiva del mondo dietro la spinta di quell' «intenerimento narcisistico» che sottosta all'urgenza autobiografica che muove la penna dell'autore. Pare dunque più che ovvio l'innesco pittorico di tali esiti letterari, o anzi, sarebbe meglio dire verbali. Le suggestioni derivanti dalle Belle Arti, d'altro canto, agiscono in qualità di reagenti sul raccordo stesso tra l'innovazione delle forme letterarie a cui tende l'autore e le ragioni pratico-poetiche che la sottendono: comportano precise scelte a livello stilistico e sintattico nel delinearsi della cifra personalissima della sua prosa. 


\section{Bibliografia}

BATTISTINI, Alberto, RAIMONDI, Ezio (1984): «Le poetiche della realtà nel periodo dell'industrializzazione», Letteratura italiana. Le forme del testo. Teoria e poesia, diretta da Asor Rosa, Torino, Einaudi: 222-236.

BIETOLETTI, Silvestra (2001): I Macchiaioli. La storia, gli artisti, le opere, Firenze, Giunti.

BIGAZZI, Roberto (1969): I colori del vero. Vent'anni di narrativa: 1860-1880, Roma-Bari, Laterza.

CAlABRESE, Omar (1985): «Visibile/leggibile», Il linguaggio dell'arte, Milano, Bompiani: 171-177.

CAPUANA, Luigi (1882): "Carlo Dossi» in Studi sulla letteratura contemporanea. Seconda serie, Catania, Giannotta: 57-72. (poi in Studi sulla letteratura contemporanea. Seconda serie, a cura di Paola Azzolino, Napoli, Liguori, 1988).

CAPUTO, Francesca (2000): Sintassi e dialogo nella narrativa di Carlo Dossi, Firenze, Accademia della Crusca.

CARNERO, Roberto, a cura di (2007): La poesia scapigliata, Milano, Rizzoli, 2007.

CONTINI, Gianfranco (1988): «Espressionismo letterario» in Ultimi esercizi ed elzeviri (1968-1987), Torino, Einaudi: 41-108.

Dossi, Carlo (1964): Note azzurre, a cura di Dante Isella, Milano, Adelphi.

Dossi, Carlo (1994): Due racconti giovanili, edizione e introduzione di Paola Montefoschi, Roma, Salerno editrice.

Dossi, Carlo (1995): Opere, a cura di Dante Isella, Milano, Adelphi.

FARINELl, Giuseppe (2003): La Scapigliatura: profilo storico, protagonisti, documenti, Roma, Carocci.

ISELlA, Dante (1958): La lingua e lo stile di Carlo Dossi, Milano-Napoli, Ricciardi.

ISELLA, Dante (1988): «Nota introduttiva» in Carlo Dossi, L'Altrieri - Vita di Alberto Pisani, Torino, Einaudi: V-XVI.

ISELLA, Dante (1995a): «La memoria dell'Altrieri» nell'introduzione a Carlo Dossi, Opere, Milano, Adelphi: XXI-XXVI (prima «Nota introduttiva» in Carlo Dossi, L'Altrieri. Nero su bianco, Torino, Einaudi, 1972: V-IX).

ISELlA, Dante (1995b): «La scrittura-labirinto» nell'introduzione a Carlo Dossi, Opere, Milano, Adelphi: XXVII-XLI (prima «Nota introduttiva» in Carlo Dossi, La Desinenza in A, Torino, Einaudi, 1981: V-XVI).

LeVI, Luigi Primo (1913): Tranquillo Cremona. L'uomo, l'artista nei ricordi di Luigi Perelli e Primo Levi L'Italico, Milano, Alfieri e Lacroix Editori.

LuCINI, Gian Pietro (1911): L'ora topica di Carlo Dossi. Saggio di critica integrale, Varese, Nicola.

MARIANI, Gaetano (1967): Storia della scapigliatura, Caltanissetta-Roma, Sciascia.

MAZZACURATI, Giancarlo (1991): «L'arte del titolo, da Sterne a Pirandello», MLN, Italian Issue, 106, 1: 38-77.

MonTEFosChI, Paola (1994): «Introduzione» in Carlo Dossi, Due racconti giovanili, Roma, Salerno editrice: 4-21.

NARDI, Piero (1968): Scapigliatura. Da Giuseppe Rovani a Carlo Dossi, Milano, Mondadori (prima, Bologna, Zanichelli, 1924). 
PATRIZI, Giorgio (2005): «Visività del comico. Note su Dossi», Il comico nella letteratura italiana. Teorie e poetiche, a cura di Silvana Cirillo, Roma, Donzelli: 259-276.

PATRIZI, Giorgio (2009): «Parola e immagine: le ragioni e le origini delle avanguardie novecentesche», Italianistica, 3: 31-39.

PozZI, Giovanni (1996/1981): La parola dipinta, Milano, Adelphi.

PORTINARI, Folco (1976): Un 'idea di realismo, Napoli, Guida: 214-240.

PORTINARI, Folco (2004): «Introduzione» a Carlo Dossi, Opere scelte, Torino, UTET.

PRAZ, Mario (1971): Mnemosine. Parallelo tra la letteratura e le arti visive, Milano, Mondadori.

RENZI, Lorenzo, a cura di (1988): Grande grammatica italiana di consultazione. La frase, vol. I, Bologna, Il Mulino.

RosA, Giovanna (1997): La narrativa degli Scapigliati, Roma-Bari, Laterza.

Rovani, Giuseppe (1874): Le Tre Arti considerate in alcuni illustri italiani contemporanei, Milano, Fratelli Treves Editori.

SACCO, Luigi (1996): «Prefazione» in Carlo Dossi, L'Altrieri. Nero su bianco, Milano, Garzanti: XVI-XXV.

SACCONE, Antonio (1995): «Pinti romanzi» e «scritte pitture», in Carlo Dossi. La scrittura del margine, Napoli, Liguori: 69-94.

SCARSI, Giovanna (1979): Scapigliatura e Novcecento: poesia, pittura, musica, Roma, Edizioni Studium. 